\title{
Water table depth prediction based on Deep learning models in Electrical Power Transmission Lines area
}

\author{
Shijun Wang ${ }^{1}$, Chang Ping ${ }^{2}$, Ning Wang ${ }^{3}$, Jing Wen ${ }^{3}$, Ke Zhang ${ }^{4}$, Kun Yuan ${ }^{5}$, and Jun \\ Yang $^{6}$ \\ ${ }^{1}$ Economic and Technical Research Institute of State Grid Gansu Province Electric Power \\ Company, LTD. \\ ${ }^{2}$ Economic and Technical Research Institute of State Grid Gansu Province Electric Power \\ Company, LTD. \\ ${ }^{3}$ China Aero Geophysical Survey and Remote Sensing Center for Land and Resources \\ ${ }^{4}$ State Grid Electric Power Research Institute Co. \\ ${ }^{5}$ State Grid Electric Power Research Institute Co., Anhui Jiyuan Electric Power System \\ Tech Co. LTD. \\ ${ }^{6}$ State Grid Zhejiang Electric Power Co Electric Power Research Institute
}

December 1, 2021

\begin{abstract}
Background: Predicting water table depth in Electrical Power Transmission Lines area presents great importance and helps the decision makers do the safety analysis during the project. The present study predicts the water table depth with observed weather data and hydrologic data. Method: The study first compared the results of LSTM, GRU, LSTM-S2S, and FFNN models in daily data simulation. Moreover, two scenarios (S1 and S2) were set to identify the effect of the water component on water table depth simulation. In addition, in order to analyze how data time scale influences the model simulation results, the monthly scale data was simulated by LSTM, GRU, and LSTM-S2S models. Result: The result indicated that LSTM-S2S was the best model for predicting daily water table depth among the four models. By contrast, FFNN performed the worst. LSTM and GRU model performed equally well both in daily data and monthly data simulation. S1 performed better than S2 in the water table depth simulation. The average daily performance of R2 and NSE was both higher than that in the monthly results with LSTM, GRU, and LSTM-S2S models. Conclusion: As a result, the method in the present study can be used to simulate the water table depth in the future in Electrical Power Transmission Lines area.
\end{abstract}

\section{Hosted file}

MANUSCRIPT.docx available at https://authorea.com/users/449022/articles/547685-water-tabledepth-prediction-based-on-deep-learning-models-in-electrical-power-transmission-linesarea

\section{Hosted file}

Figures.pdf available at https://authorea.com/users/449022/articles/547685-water-tabledepth-prediction-based-on-deep-learning-models-in-electrical-power-transmission-linesarea 